\title{
The First $\left\{\mathrm{Dy}_{4}\right\}$ Single-Molecule Magnet with a Toroidal Magnetic Moment in the Ground State
}

\author{
Peng-Hu Guo, ${ }^{\dagger}$ Jun-Liang Liu, ${ }^{\dagger}$ Ze-Min Zhang, ${ }^{\dagger}$ Liviu Ungur, ${ }^{\dagger}$ Liviu F. Chibotaru, ${ }^{*}$, Ji-Dong Leng, $^{\dagger}$ \\ Fu-Sheng Guo, ${ }^{\dagger}$ and Ming-Liang Tong*,
}

${ }^{\dagger}$ MOE Key Lab of Bioinorganic and Synthetic Chemistry, State Key Laboratory of Optoelectronic Materials and Technologies, School of Chemistry \& Chemical Engineering, Sun Yat-Sen University, Guangzhou 510275, People's Republic of China

${ }^{\ddagger}$ Division of Quantum and Physical Chemistry and Institute of Nanoscale Physics and Chemistry (INPAC), Celestijnenlaan 200F, Katholieke Universiteit Leuven, Leuven B-3001, Belgium

\section{Supporting Information}

ABSTRACT: A toroidal magnetic moment in the absence of a conventional total magnetic moment was first observed in a novel tetranuclear dysprosium cluster with nonmagnetic ground state. The toroidal state is quite robust with respect to variations of the exchange parameters.

$\mathrm{W}$ ith consideration of the potential applications in new storage and information-processing technologies, the researches on single-molecule magnets (SMMs) are recognized as an important area for molecular magnetism. ${ }^{1}$ Thanks to the large intrinsic magnetic anisotropy of lanthanide ( $\mathrm{Ln}$ ) ions, remarkable efforts have been focused on the pure $4 \mathrm{f}$ SMMs and single-ion magnets (SIMs) in recent years. ${ }^{2-7}$ For the SMMs incorporating multiple $\mathrm{Ln}$ ions, though weak magnetic interactions, the very large anisotropy favors the orientation of magnetization along an easy axis. The anisotropic barrier of an SMM is derived from a combination of both the single ionic anisotropy and exchange interaction in these complexes, ${ }^{3 \mathrm{~b}, 6 \mathrm{~b}}$ while the zero-field splitting on Ln sites is much larger than the exchange splitting. Dy ${ }^{\mathrm{III}}$ is one of the most attractive heavy rareearth ions showing the following unique features: first, Dy ${ }^{\mathrm{II}}$ ions will always maintain a high-magnitude $\pm m_{J}$ quantum number and a doubly degenerate ground state (ground Kramers' doublet) because of its odd electron count; second, a relatively large separation between the bistable ground state and the first excited state relaxes the spin much slower. ${ }^{2}$

Since the first case was revealed in the famous $\left\{\mathrm{Dy}_{3}\right\}$ triangle, detection of the other lanthanide molecular species with toroidal magnetic moments has become an interesting topic of lanthanide-based SMM chemistry. ${ }^{2,3}$ The noncollinearity of the single-ion easy axes of magnetization of the Dy ${ }^{\mathrm{III}}$ ions leads to a nonmagnetic ground state, giving rise to the toroidal magnetic moments on the Dy ${ }^{\mathrm{III}}$ sites and also the slow relaxation behavior. ${ }^{6}$ Herein we report a novel $\left\{\mathrm{Dy}_{4}\right\}$ SMM, $\left[\mathrm{Dy}_{4}\left(\mu_{3}-\right.\right.$ $\left.\mathrm{OH})_{2}(\mu-\mathrm{OH})_{2}(2,2-\mathrm{bpt})_{4}\left(\mathrm{NO}_{3}\right)_{4}(\mathrm{EtOH})_{2}\right][\mathbf{1} ; 2,2-\mathrm{bptH}=3,5-$ bis(pyridin-2-yl)-1,2,4-triazole], which is the first case of the $\left\{\mathrm{Dy}_{4}\right\}$ family with a toroidal magnetic moment in the ground state.

Colorless brick crystals of 1 were obtained from solvothermal reactions of $\mathrm{Dy}\left(\mathrm{NO}_{3}\right)_{3} \cdot 5 \mathrm{H}_{2} \mathrm{O}$ with 2,2-bptH in the presence of triethylamine. Notably, the $\mathrm{bpt}^{-}$ligand with two bidentate chelating sites is, for the first time, used to produce $\mathrm{Ln}^{\mathrm{III}}$ complexes because all of the reported bpt-based compounds are transition-metal complexes of $\mathrm{Cu}^{\mathrm{I}}, \mathrm{Fe}^{\mathrm{II}}, \mathrm{Co}^{\mathrm{II}}$, and $\mathrm{Ni}^{\mathrm{II}} .8$

Single-crystal X-ray crystallography (see the Supporting Information, SI) reveals that the centrosymmetric complex 1 has four precisely coplanar Dy ${ }^{\text {III }}$ ions (Figure 1) with the

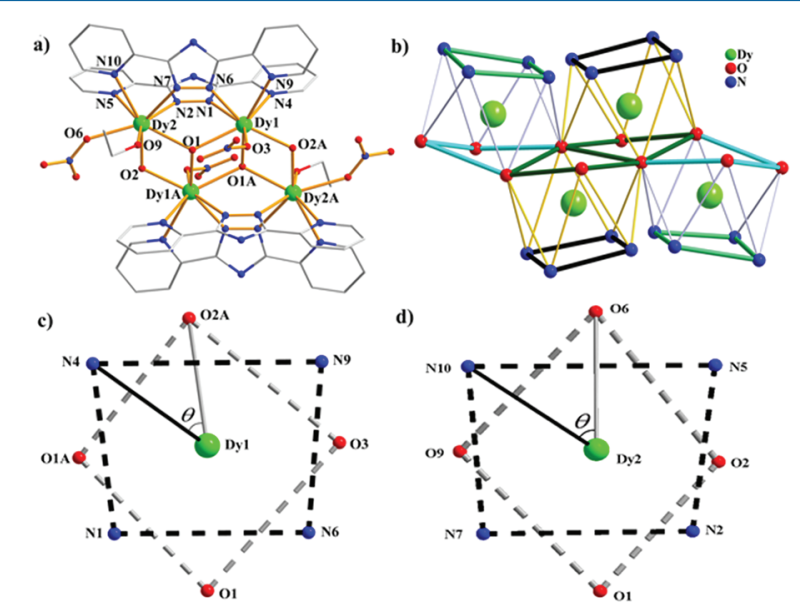

Figure 1. Molecular (a) and core (b) structures of 1. SAP environment with skew angle $\theta$ between the diagonals of the two squares (c and d).

Dy...Dy distances of 3.73-4.28 Å. Each eight-coordinate Dy ${ }^{\mathrm{III}}$ ion possesses a distorted square-antiprismatic (SAP) geometry with a $\mathrm{N}_{4} \mathrm{O}_{4}$ coordination environment like in Ishikawa's SIMs $\left[\mathrm{Pc}_{2} \mathrm{Ln}\right] .^{7}$ The skew angles $\theta$ range from $37.2^{\circ}$ to $53.2^{\circ}$, indicating that the coordination sphere is more slightly distorted from the ideal SAP geometry (skew angle is $\left.45^{\circ}\right)^{7}$ than that of 1 . All Dy ${ }^{\mathrm{III}}$ ions are the vertices of the parallelogram bridged by two $\mu_{3}-\mathrm{OH}$, two $\mu-\mathrm{OH}$, and four $\mu$-bpt ligands, two of whose $\mu_{3} \mathrm{OH}$ are located on the opposite sides of the $\left\{\mathrm{Dy}_{4}\right\}$ plane. By comparison, two similar $\left\{\mathrm{Dy}_{4}\right\}$ clusters with similar planar central cores surrounded by the $\mathrm{N}, \mathrm{O}$-containing ligands have recently been reported, ${ }^{4 a}$ in which the $\mathrm{Dy}^{\mathrm{III}}$ ions are coordinated in the distorted $\mathrm{O}_{6} \mathrm{~N}_{2}$ and $\mathrm{O}_{6} \mathrm{Cl}_{2}$ or $\mathrm{O}_{5} \mathrm{~N}_{2} \mathrm{Cl}$ SAP geometry.

Received: December 9, 2011

Published: January 12, 2012 
The $\chi_{\mathrm{M}} T$ value at $300 \mathrm{~K}$ is $56.4 \mathrm{~cm}^{3} \mathrm{~K} \mathrm{~mol}^{-1}$, which is in good agreement with the expected value of $56.7 \mathrm{~cm}^{3} \mathrm{~K} \mathrm{~mol}^{-1}$ for four uncoupled Dy ${ }^{\mathrm{III}}$ ions $\left(S={ }^{5} / 2, L=5,{ }^{6} H_{15 / 2}\right.$, and $g=$ $\left.{ }^{4} /{ }_{3}\right){ }^{9}$ Upon lowering of the temperature, the $\chi_{M} T$ values gradually decrease to $20.5 \mathrm{~cm}^{3} \mathrm{~K} \mathrm{~mol}^{-1}$ for 1 at $2.0 \mathrm{~K}$, suggesting the progressive depopulation of excited-state Stark sublevels due to the crystal-field effects of $\mathrm{Dy}{ }^{\mathrm{III}}$ and/or antiferromagnetic interactions. ${ }^{7,9}$ The field dependence of magnetization (Figure 2, inset, and Figure S1 in the SI) rises

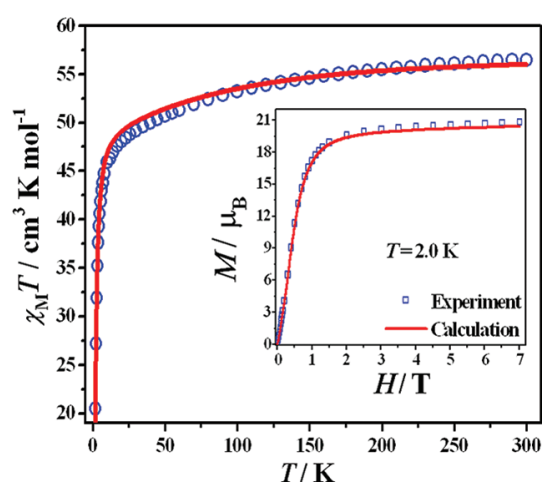

Figure 2. Comparison between the measured magnetic susceptibility (empty circles) and calculated (red line) magnetic susceptibility for $\mathbf{1}$. Inset: Comparison between the measured (empty squares) and calculated (red line) molar magnetization at $2.0 \mathrm{~K}$ for 1 .

abruptly at low fields before leveling to $19.2 \mu_{\mathrm{B}}$ around $15 \mathrm{kOe}$ at $2.0 \mathrm{~K}$, in good agreement with the expected value $(4 \times 5.23$ $\mu_{\mathrm{B}}$ ) for four isolated $\mathrm{Dy}{ }^{\mathrm{III}}$ ions, and then continues to increase slowly as in other reported Dy ${ }^{\text {III }}$ systems as a result of the significant anisotropy. ${ }^{4 a, b}$

In order to gain insight into the local electronic and magnetic properties, we have performed high-level ab initio calculations on individual Dy centers (Figures S2 and S3 in the SI) with the MOLCAS 7.6 program package (see the SI for computational details, energies of the local $\mathbf{g}$ tensors of the ground Kramers' doublets on $\mathrm{Dy}{ }^{\mathrm{III}}$ sites, and relative angles between the main anisotropy axes). ${ }^{10}$ It was found that the ground Kramers' doublet on each of the two nonequivalent Dy sites is well separated from the first excited one (Table S1 in the SI), while the main values of the $\mathbf{g}$ tensors of the lowest doublets on Dy ${ }^{\mathrm{III}}$ sites obtained are very axial, which is probably a result of the $\mathrm{N}_{4} \mathrm{O}_{4}$ coordination geometries and close to a square antiprism. The main anisotropy axes of the ground Kramers' doublets on Dy ${ }^{\text {III }}$ sites in $\mathbf{1}$ are shown in Figure S4 in the SI by dashed lines. The four axes form an almost perfect parallelogram and lie practically in the $\mathrm{Dy}_{4}$ plane.

The magnetic interactions between Dy ions in $\mathbf{1}$ include contributions from magnetic dipole-dipole and exchange interactions. The exchange coupling was simulated within the Lines model as described elsewhere. ${ }^{3}$ The dipolar interaction between Dy ions was calculated on the basis of the local $\mathbf{g}$ tensors of the corresponding ground Kramers' doublets obtained in ab initio calculations (Tables S2 and S3 in the SI). When projected on the ground Kramers' doublets of the four Dy ions, the magnetic interaction takes the form of a noncollinear Ising Hamiltonian (Figure S5 in the SI):

$$
\begin{aligned}
& H=-J_{1}\left(s_{1, z_{1}} s_{2^{\prime}, z_{2}{ }^{\prime}}+s_{1^{\prime}, z_{1},} s_{2, z_{2}}\right)
\end{aligned}
$$

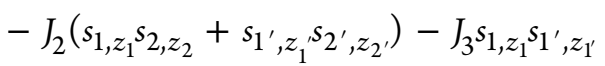

$$
\begin{aligned}
& -J_{4} s_{2, z_{2}} s_{2^{\prime}, z_{2}^{\prime}}
\end{aligned}
$$

where $s_{i, z_{i}}$ are projection operators of the effective spin $s=1 / 2$ of $\mathrm{Dy}_{i}$ on the corresponding anisotropy axis $z_{i}$ (Figure S5 in the SI). $J_{i}$ are the parameters of the total magnetic interaction:

$$
J_{i}=J_{i}^{\text {dipolar }}+J_{i}^{\text {exch }}
$$

where $J_{i}^{\text {dipolar }}$ is the dipolar contribution and $J_{i}^{\text {exch }}$ is the exchange contribution. Table 1 shows the calculated $J_{i}^{\text {dipolar }}$ and fitted $J_{i}^{\text {exch }}$

Table 1. Parameters of the Magnetic Interactions between Dy Ions in 1 (in $\mathrm{cm}^{-1}$ )

\begin{tabular}{lrrrc} 
& \multicolumn{1}{c}{$J_{1}$} & \multicolumn{1}{c}{$J_{2}$} & $J_{3}$ & $J_{4}$ \\
$J_{\mathrm{i}}^{\text {dipolar }}$ & 5.06 & 1.71 & 2.64 & 0.42 \\
$J_{\mathrm{i}}^{\text {exch }}$ & -0.62 & -0.49 & 0.45 & 0.00 \\
$J_{i}$ & 4.44 & 1.22 & 3.09 & 0.42 \\
\hline
\end{tabular}

parameters, which describe the interaction between all Dy-Dy pairs in 1.

A comparison of the measured and calculated magnetic susceptibility times temperature and powder magnetization $(M)$ versus applied field $(H)$ is shown in the inset of Figure 2. We note that the only fitting parameters are the three $J_{i}^{\text {exch }}$, which are obtained much smaller than $J_{i}^{\text {dipolar }}$ (Table 1), as is generally expected. The spectrum of the lowest exchange levels calculated with eq 1 is given in Table S4 in the SI. The ground exchange state is nonmagnetic and corresponds to a toroidal arrangement of local magnetic moments on Dy ${ }^{\mathrm{III}}$ sites (Figure $3)$. That the $\chi_{M}{ }^{\prime} T$ value $\left[\chi_{M}{ }^{\prime}\right.$, in-phase signals of the alternatingcurrent (ac) susceptibilities, $\left.H_{\mathrm{dc}}=0, H_{\mathrm{ac}}=5 \mathrm{Oe}\right]$ drops to nearly 0 also indicates the nonmagnetic ground state (Figure S8 in the SI). Compared with $\left\{\mathrm{Dy}_{3}\right\}$ triangles, ${ }^{3}$ where also a large toroidal moment was observed in the ground state, the present compound, $\mathbf{1}$, is the first example showing a strong toroidal magnetization in the absence of a conventional total magnetic moment. We stress that the toroidal ground state is quite robust with respect to variations of the exchange parameters; for instance, it is stabilized already for $J_{i}^{\text {exch }}=0$, i.e., by dipolar interactions alone.

The local magnetic moments on Dy ${ }^{\mathrm{III}}$ sites in excited exchange states are shown in Figure 3. We can see that the first excited exchange doublet possesses already the largest magnetic moment (doublet 2 in Table S4 in the SI). Given the relatively small energy separation from the ground doublet state (Figure S7 in the SI), its large magnetic moment explains why the lowtemperature $\chi_{\mathrm{M}} T$ and low-field $M(H)$ do not show a pronounced $S$ shape.

To investigate the dynamics of the magnetization, ac susceptibility studies were undertaken for 1 (Figures 4 and $S 8$ in the SI). Frequency-dependent out-of-phase $\left(\chi_{M}{ }^{\prime \prime}\right)$ signals in the ac susceptibilities are indicative of slow relaxation of the magnetization associated with SMM behavior. Note that they correspond to the slowing of the reversal of the magnetic moment in the first excited doublet state (Figure 3) because the ground doublet state is nonmagnetic, as discussed above. The ac susceptibilities, along with Cole-Cole plots showing quasisemicircles and fitted by the generalized Debye function 


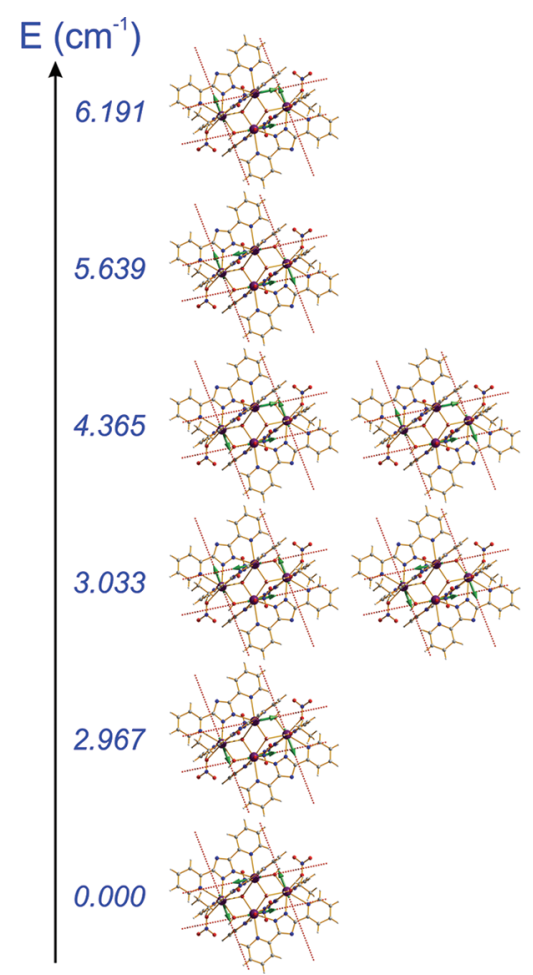

Figure 3. Orientations of the local magnetic moments shown for one of the two components of each exchange doublet state in $\mathbf{1}$. The second component of each doublet corresponds to the reversal of the magnetic moments on Dy ions (cf. Figure S6 in the SI). The numbers on the vertical axis are the calculated exchange energies (see Table S4 in the SI).

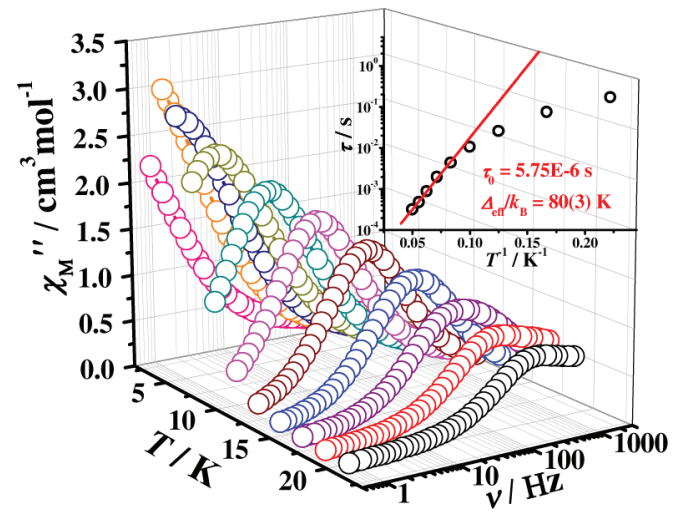

Figure 4. Out-of-phase susceptibility $\chi^{\prime \prime}$ versus frequency $\nu$ with 0 Oe $\mathrm{dc}$ field and $5 \mathrm{Oe}$ ac field at frequencies between 1 and $1500 \mathrm{~Hz}$ in the logarithmic scale for a polycrystalline sample of $\mathbf{1}$ in the temperature range $1.8-20 \mathrm{~K}$. Inset: Magnetization relaxation time $(\tau)$ versus $T^{-1}$ plot for 1. The solid line corresponds to the Arrhenius law.

(Figure S9 in the SI; $\alpha=0.14-0.22),{ }^{11}$ indicate the presence of a relatively narrow width of the distribution of slow relaxation. The effective energy barrier, $\Delta_{\text {eff }}$ can be obtained from the high-temperature regime of the relaxation time by the Arrhenius law, giving $\Delta_{\text {eff }} / k_{\mathrm{B}}=80(3) \mathrm{K}$ and the preexponential factor $\tau_{0}=5.75 \times 10^{-6} \mathrm{~s}$ (inset of Figure 4). At lower temperatures, the relaxation time increases much more slowly with decreasing temperature, suggesting the onset of a quantum tunneling regime. The effective energy barriers derived from the relaxation times are 1 order of magnitude larger than the spread of exchange levels in $\mathbf{1}$ (see Figure 3), which means that they arise from magnetic blocking on the local Dy sites rather than from exchange interaction.

In summary, we have investigated the first $\left\{\mathrm{Dy}_{4}\right\}$ SMM whose nonmagnetic ground exchange state corresponds to a toroidal arrangement of local magnetic moments on Dy ${ }^{\mathrm{III}}$ sites calculated by ab initio investigation.

\section{ASSOCIATED CONTENT}

S Supporting Information

Experimental details, X-ray crystallography, ab initio calculation of the magnetism, ac susceptibility data, and CCDC 848450. This material is available free of charge via the Internet at http://pubs.acs.org.

\section{AUTHOR INFORMATION}

\section{Corresponding Author}

*E-mail: tongml@mail.sysu.edu.cn (M.-L.T.), Liviu. Chibotaru@chem.kuleuven.be (L.F.C.).

\section{ACKNOWLEDGMENTS}

This work was supported by the NSFC (Grants 91122032, 50872157, 90922009, and 20821001) and the "973 Project" (Grant 2012CB821704). L.U. gratefully acknowledges the research grant Methusalem from Katholieke Universiteit Leuven.

\section{REFERENCES}

(1) Gatteschi, D.; Sessoli, R. Angew. Chem., Int. Ed. 2003, 42, 268.

(2) (a) Sessoli, R.; Powell, A. K. Coord. Chem. Rev. 2009, 253, 2328. (b) Gatteschi, D. Nat. Chem. 2011, 3, 830. (c) Rinehart, J. D.; Long, J. R. Chem. Sci. 2011, 2, 2078.

(3) (a) Tang, J.; Hewitt, I.; Madhu, N. T.; Chastanet, G.; Wernsdorfer, W.; Anson, C. E.; Benelli, C.; Sessoli, R.; Powell, A. K. Angew. Chem., Int. Ed. 2006, 45, 1729. (b) Chibotaru, L. F.; Ungur, L.; Soncini, A. Angew. Chem., Int. Ed. 2008, 47, 4126.

(4) (a) Lin, P.-H.; Burchell, T. J.; Ungur, L.; Chibotaru, L. F.; Wernsdorfer, W.; Murugesu, M. Angew. Chem., Int. Ed. 2009, 48, 9489. (b) Yan, P.-F.; Lin, P.-H.; Habib, F.; Aharen, T.; Murugesu, M.; Deng, Z.-P.; Li, G.-M.; Sun, W.-B. Inorg. Chem. 2011, 50, 7059. (c) Zheng, Y.-Z.; Lan, Y.; Anson, C. E.; Powell, A. K. Inorg. Chem. 2008, 47, 10813. (d) Guo, Y.-N.; Xu, G.-F.; Gamez, P.; Zhao, L.; Lin, S.-Y.; Deng, R.; Tang, J.; Zhang, H.-J. J. Am. Chem. Soc. 2010, 132, 8538.

(5) Guo, F.-S.; Liu, J.-L.; Leng, J.-D.; Meng, Z.-S.; Lin, Z.-J.; Tong, M.-L.; Gao, S.; Ungur, L.; Chibotaru, L. F. Chem.-Eur. J. 2011, 17, 2458.

(6) (a) Lin, P.-H.; Burchell, T. J.; Clérac, R.; Murugesu, M. Angew. Chem., Int. Ed. 2008, 47, 8848. (b) Guo, Y.-N.; Xu, G.-F.; Wernsdorfer, W.; Ungur, L.; Guo, Y.; Tang, J.; Zhang, H.-J.; Chibotaru, L. F.; Powell, A. K. J. Am. Chem. Soc. 2011, 133, 11948. (c) Hewitt, I. J.; Tang, J.; Madhu, N. T.; Anson, C. E.; Lan, Y.; Luzon, J.; Etienne, M.; Sessoli, R.; Powell, A. K. Angew. Chem., Int. Ed. 2010, 49, 6352. (d) Blagg, R. J.; Muryn, C. A.; Mclnnes, E. J. L.; Tuna, F.; Winpenny, R. E. P. Angew. Chem., Int. Ed. 2011, 50, 6530. (e) Habit, F.; Lin, P.-H.; Long, J.; Korobkov, I.; Wernsdorfer, W.; Murugesu, M. J. Am. Chem. Soc. 2011, 133, 8830.

(7) Jiang, S.-D.; Wang, B.-W.; Sun, H.-L.; Wang, Z.-M.; Gao, S. J. Am. Chem. Soc. 2011, 133, 4730.

(8) (a) Bao, X.; Leng, J.-D.; Meng, Z.-S.; Lin, Z.-J.; Tong, M.-L.; Nihei, M.; Oshio, H. Chem.-Eur. J. 2010, 16, 6169.

(9) Kahn, O. Molecular Magnetism; VCH Publishers: New York, 1993.

(10) Chibotaru, L.; Ungur, L. Computer programs SINGLE_ANISO and POLY_ANISO; University of Leuven: Leuven, Belgium, 2006.

(11) Cole, K. S.; Cole, R. H. J. Chem. Phys. 1941, 9, 34. 